\title{
Bi-directional Full-bridge DC-DC Converters with Phase-shifting Control and Its Phase-shifting Range Analysis
}

\author{
Jiabo Yuan ${ }^{1,}$ a, Xin Wang ${ }^{1, b^{*}}$, Yihui Zheng ${ }^{1}$, Lixue $\mathrm{Li}^{1}$, Daqian Shen ${ }^{2}$, Quanlun \\ Deng $^{2}$ \\ ${ }^{1}$ Center of Electrical \& Electronic Technology, Shanghai Jiao Tong University, Shanghai, China \\ ${ }^{2}$ Guang'an Power Supply Company of State Grid Sichuan Electric Power Company, China \\ ayetash@sjtu.edu.cn, bwangxin26@sjtu.edu.cn
}

Keywords: Phase-shifting control, bi-directional full-bridge DC-DC converters, soft switching, phase-shifting angle.

Abstract. In order to simplify the generation of phase-shifting waveform and reduce the range of phase angle, an angel-fixed method to generate the phase-shifting waveform is presented in this paper. Firstly, it studies the advantages and the topology structure of the bi-directional full-bridge DC-DC converter, compares the advantages and disadvantages of the phase-shifting control and DC voltage variable control, and analyzes the principle of the phase-shifting control. So the soft switching conditions and the transmission characteristics can be concluded. Then, a new method of generating phase-shifting waveform is designed, which is easy to change phase-shifting angle. The method above fixes the waveform of primary side and can only change the waveform phase angel of the secondary side. So it is more convenient to change the phase-shifting angle and beneficial to reduce the transmission power consumption. Moreover, due to the generation of fixed waveform, the phase-shift angle varies in the range of 90 degrees, which is conducive to the realization of soft switching. Finally, the practical experiment proves that it can not only realize the soft switching but also reduce the transmission power consumption.

\section{Introduction}

With the popularization of photovoltaic generation technology, photovoltaic grid-connected system has become the main trend. Compared with the former system, the photovoltaic grid-connected system with the energy storing meet the needs of family and business users better [1,2].

Bi-directional full-bridge DC-DC converter has two control modes. DC variable voltage control, that is, invert $\mathrm{DC}$ to $\mathrm{AC}$ by the full-bridge, then raise the $\mathrm{AC}$ voltage by the transformer, and convert $\mathrm{AC}$ to DC. Because AC voltage can only be lower than the DC voltage in the inverter, the power ratio is determined by the transformer and the range is limited [3]. The phase-shifting control can produce a square wave signal with a phase-shifting in the primary and secondary side of the bi-directional full-bridge circuit by controlling the driving pulse of two full-bridge. The power and transmission direction can be adjusted by controlling the phase-shifting angle. The phase-shifting control has not only higher transformer ratio compared with DC variable control, but also can realize soft switching, and have small system inertia and fast dynamic response [4].

In this paper, an angel-fixed method to generate the phase-shifting waveform is designed. First, it introduces the topology structure of the bi-directional full-bridge DC-DC converter, then it analyzes the principle of phase-shifting control. Secondly, it concludes the influences of phase-shifting angel on the soft switching and the transmission power. Based on the study of the soft switching conditions and transmission characteristics, the angel-fixed method is designed, it is more convenient to change the phase-shifting angel and beneficial to reduce the transmission power loss. Finally, the method is applied in practical experiment and realizes the soft switching and reduces the power consumption. 


\section{Bi-directional Full-bridge DC-DC Converter}

Full-bridge DC-DC converter voltage stress is smaller, and is suitable for high power applications. The bi-directional full-bridge DC-DC converter adds a power transistor on the diode, so that secondary side can also control the switch, as shown in Fig. 1.

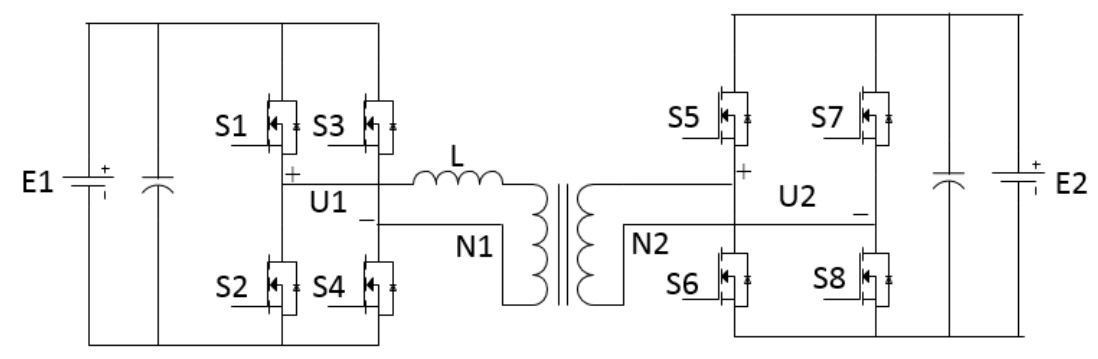

Fig. 1. Bi-directional Fullbridge Converter

\section{The Characteristics of Phase-shifting Control}

Transmission Power Analysis. According to the analysis of second section, inductance current can be changed by the phase-shifting angel, so that we can control the direction and size of the power on bi-directional full-bridge, the lead square wave is output direction, the lag square wave is the direction of the input, the transmission direction of the circuit is from lead square wave to the lag square wave. Fig. 2 shows the relationship between phase-shifting angle and transmission power.

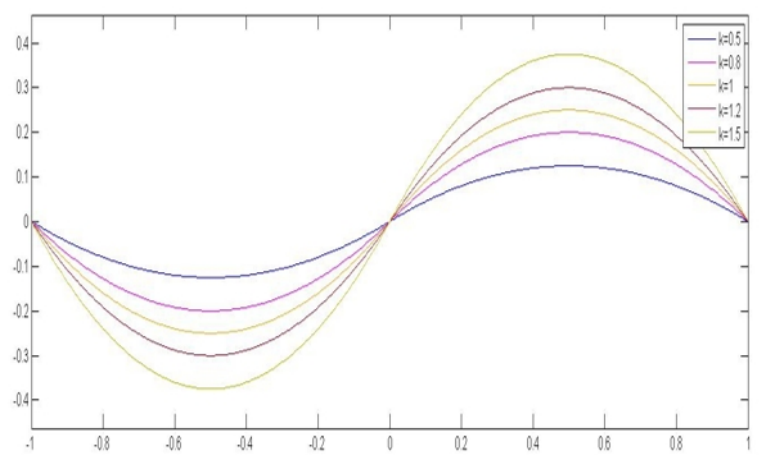

Fig. 2. Relationship of Phase-shifting Angle and Transmission Power

The curve also shows that the transmission power is the maximum when the $\mathrm{D}=0.5$ and -0.5 . When the phase angle is 0 degrees and 180 degrees, the power transmission is 0 .

Soft Switching Analysis. According to the work condition, when the inductor current is negative, the inductance current flows through the diode of the S1 and S4. At this time switches close, switch voltage is only diode forward voltage, which realizes zero voltage switching. On the secondary side, when the inductance current is negative, the current of the secondary side flows through the diode of S5 and S8. At this time switches close, switch voltage is diode forward voltage, which realizes zero voltage switching.

Therefore, in order to realize the zero voltage switching on both primary side and secondary side. The inductor current at the $\mathrm{t}_{0}$ time is negative, at $\mathrm{t}_{1}$ time is positive, which is shown as

$$
\frac{1}{2}\left(1-\frac{1}{k}\right) \leq D \leq \frac{1}{2}, \frac{1}{2}(1-k) \leq D \leq \frac{1}{2},
$$

According to Eq. 1, Fig. 3 can be obtained. From the Fig. 3 the soft switching ranging from the dotted line to the solid line. 


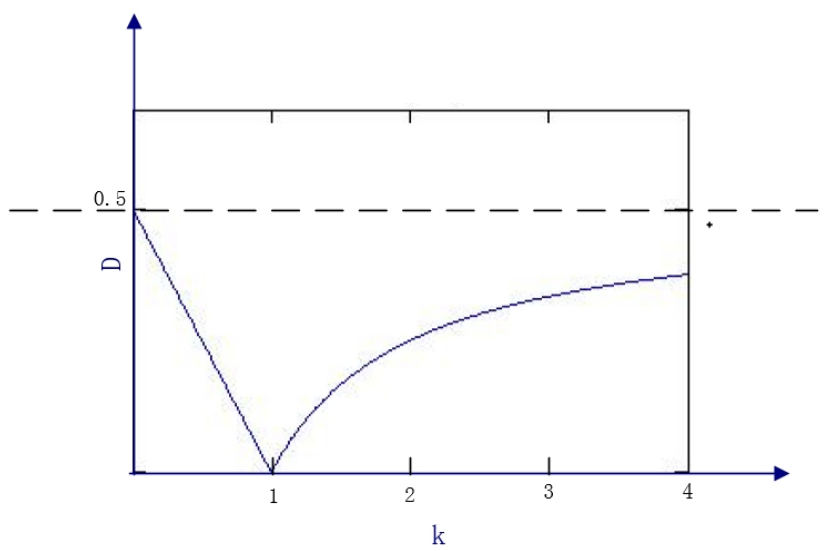

Fig. 3. Soft Switching

The Generation of Phase-shifting Wave. The generation of the phase-shifting wave is to use each counter with comparators CMPA and CMPB. When the counter rises to the setting value of CMPA, the output waveform becomes positive. When the counter declines to the setting value of CMPB, the output waveform becomes negative. So the square wave of one side can be got.

By setting the comparators for two different values, we can get the square waves of different phases, as long as the value of CMPB2-CMPB1 is equal to the value of CMPA2-CMPA1, but this method needs to change the two phases of square waves, which is complicated. Setting the value of CMPA1 and CMPB1 for the midpoint, the phase-shifting angel can be changed by the CMPA2 and CMPB2, as shown in Fig. 4.

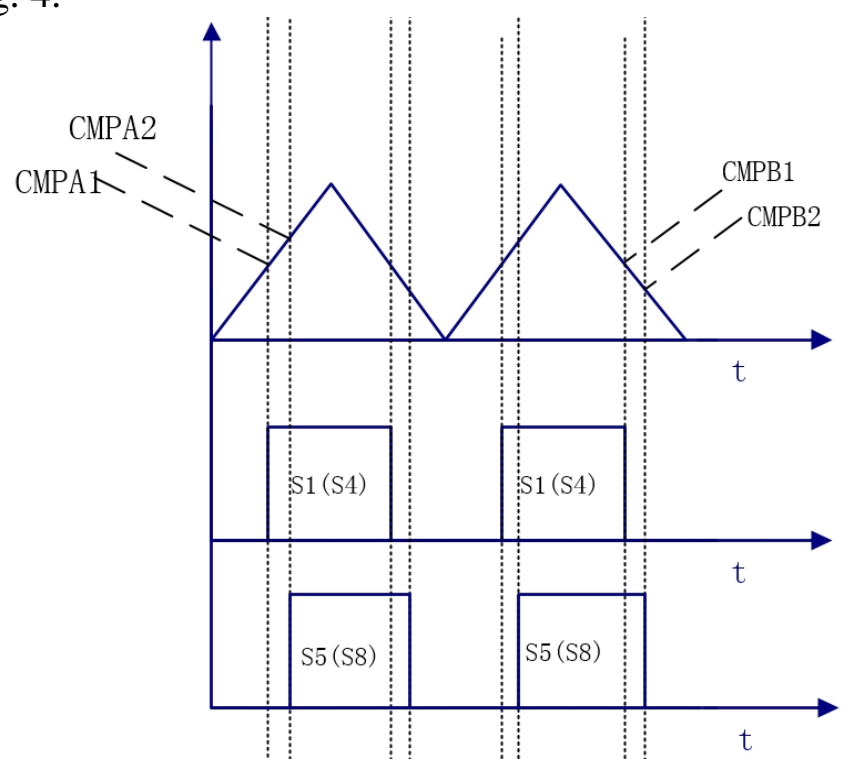

Fig. 4. Generation of Phase-shifting Square Wave

Because the comparators of switch S1 set value for the midpoint, so the maximum difference value between the comparators of the switch S5 and S1 is 1/4T, in which the maximum phase-shifting angle is 90 degrees.

\section{The Experiment Analysis}

Experiments are carried out with load. The phase-shifting angle of the experiment is 60 degrees. In this case, both primary and secondary side switch realizes ZVS, as shown in Fig. 5. 


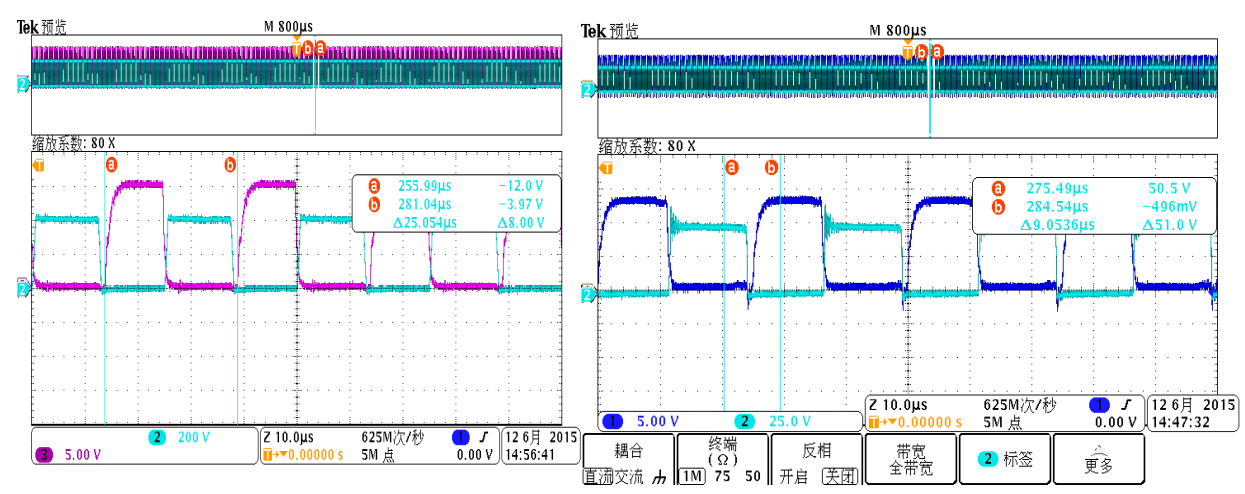

Fig. 5. Soft Switching

\section{Conclusions}

In this paper an angel-fixed method of generating the phase-shifting waveform is designed. First, the bi-directional DC-DC full-bridge converter is introduced, compared with other structures, its voltage stress is smaller and it is suitable for high power applications. Second, based on the analysis of the principle of phase-shifting control, it concludes the soft switching conditions and transmission power consumption, to realize the soft switching, the phase-shifting angel should be lower than 90 degrees. Then the angel-fixed method is designed, the new method fixes the waveform of primary side and can only change the waveform phase angel of the secondary side, which is more convenient to change the phase-shifting angle. Moreover, due to the phase-shifting angle within 90 degrees, it is beneficial to reduce the power consumption of the system and realize the soft switching. Finally, the practical experiment verifies the method above can not only realize the soft switching, but also reduce the transmission power consumption.

\section{Acknowledgements}

This work is supported by the Key Project of NSFC (No. 61533012), the Shanghai Natural Science Foundation (14ZR1421800), the State Key Laboratory of Synthetical Automation for Process Industries.

\section{References}

[1] T. Georgios, M. Georgios, Solar energy systems potential for nearly net zero energy residential buildings, J. Solar Energy. 115 (2015) 743-756.

[2] A.J. Kirkpatrick, L.S. Bennear, Promoting clean energy investment: An empirical analysis of property assessed clean energy, J. Journal of Environmental Economics and Management. 68 (2014) 357-375.

[3] B. Zhao, G.Q. Yu, W.X. Sun, Bi-directional full-bridge DC-DC converters with dual-phase-shifting control and its backflow power characteristic analysis, J. Proceedings of the CSEE. 32 (2012) 43-50.

[4] H. Cheng, Q.M. Gao, J.B. Zhu, X.K. Yang, C. Wang, Dynamic modeling and minimum back flow power controlling of the bi-directional full-bridge DC-DC converters based on dual-phase-shifting control, J. Transactions of China Electrotechnical Society. 29 (2014) 245-253.

[5] C. Wang, G.L. Sha, J. Wang, Y. Zhuang, H. Cheng, The analysis of zero voltage switching dual active bridge DC-DC converters based on dual-phase-shifting control, J. Transactions of China Electrotechnical Society. 30 (2015) 106-113.

[6] H. Zhu, H.L. Zhou, L.L. Ren, L. Gu, K. Jin, A three-phase bi-directional DC-DC converter based on phase-shifting control, J. Transactions of China Electrotechnical Society. 30 (2015) 39-46. 